\title{
The effectiveness on health education to prevent nipple trauma from breastfeeding: a systematic review
}

\section{Flávia Silva Oliveira 1}

iD https://orcid.org/0000-0003-2456-5930

Flaviana Vieira 2

iD https://orcid.org/0000-0002-3085-9428

Jessica Oliveira Cecilio 3

https://orcid.org/0000-0003-2518-7484

Janaína Valadares Guimarães 4

https://orcid.org/0000-0003-1012-4405
Suzanne Hetzel Campbell 5

iD https://orcid.org/0000-0002-8134-0669

1-4 Faculdade de Enfermagem. Universidade Federal de Goias. Rua 227 Quadra 68, s.n. Setor Leste Universitário. Goiania, GO, Brasil. CEP: 74.605-220. E-mail: flavia04silva@yahoo.com.br

5 Faculty of Nursing. University of British Columbia. Vancouver, Canada.

\begin{abstract}
Objectives: to analyze the effectiveness on health education to prevent nipple trauma in breastfeeding compared to other interventions.

Methods: systematic literature review was carried out in January 2019, according to PRISMA recommendations. The searches were conducted in Cinahl, PubMed, Web of Science, Scopus, and in the references cited in the selected articles. The studies were assessed for quality and level of evidence according to the Grading of Recommendations, Assessment, Development, and Evaluation (GRADE).

Results: twelve studies were selected, all conducted at the maternities. The interventions were classified in two categories: health education on breastfeeding and the use of nipple covers. Health education on breastfeeding presents a moderate level of evidence on the prevention of nipple trauma. There is a moderate to high level of evidence on the positive effects of guaiazulene, gel or peppermint water applied in the postpartum period to prevent nipple trauma.

Conclusion: health education on breastfeeding with clinical demonstration is an important strategy to prevent nipple trauma and requires more than one educational approach. The use of nipple covers requires some consideration, since they must be removed prior to breastfeeding. All interventions were conducted in the postpartum period, which points out for the necessity of clinical research to prevent nipple trauma in prenatal care.
\end{abstract}

Key words Breastfeeding, Wounds and injuries, Health education, Systematic review 


\section{Introduction}

Breastfeeding is the safest and most effective and economical way to feed babies. ${ }^{1}$ For mothers, it is considered a form of preventing postpartum hemorrhage and breast and ovarian cancer. ${ }^{2}$ For babies, in turn, breast milk has the ideal nutritional composition; it supports the development of bones and facial muscles, and prevents food allergies, infections, and intestinal colic, among many other benefits. ${ }^{3}$

However, the prevalence of breastfeeding is below the recommendation of the World Health Organization. ${ }^{3}$ In low and middle-income countries, an estimate of only $37 \%$ of the babies are exclusively breastfed until the age of six months. 4 Difficulties in post-discharge breastfeeding, lack of previous experience with breastfeeding, strict breastfeeding schedules, use of pacifiers, and nipple injuries are the variables associated with the interruption of exclusive breastfeeding (EBF) in the first month of lactation. 5,6

The postpartum period is recognizably a difficult time for mother and baby, and it is precisely in the first weeks of the puerperium that the main complications of the breast occur. Although it is quite common, nipple pain and trauma should not be neglected. Nipple trauma has a higher incidence between the second and third days of postpartum, 7,8 and occurs in about $26 \%$ to $52 \%$ of the women. ${ }^{9}$ It is characterized by erythema, edema, cracks, fissures, blisters, abrasions, and bruises. It is defined as a change in the normal anatomy of the nipple skin, with intrinsic primary lesion and changes in the thickness and coloration of the skin, and not merely as a solution of continuity. 10

Nipple trauma is one of the main causes of early interruption of breastfeeding 11 and, although the overall awareness on this regard, it is still a clinical problem for breastfeeding. The literature presents several forms of treating nipple trauma, including the use of lanolin, mint cream, dexpanthenol, cold and hot compresses, breast milk, breast shells, glycerin, nipple protectors, correcting latching and positioning of the baby during feeding, among others 12 ; however, the prevention of nipple trauma has not been consolidated.

The main cause of nipple trauma is poor latch by the baby and improper positioning between mother and baby. 13 Thus, health education on breastfeeding plays an important role in minimizing problems related to breastfeeding. $9,14,15$

The performance of health workers in the prevention of nipple trauma is still a challenge 16 and a scientific basis from the best evidence is needed to support decision-making in clinical practice.

There is a review in the literature that address this subject; however, it is outdated by more than five years ${ }^{17}$ and does not configure a systematic review which has gathered evidence strong enough to support clinical practice. Although another study has been recently published that configures a systematic review, it addresses prevention and treatment of nipple pain and nipple trauma at the same time, and does not have specificity and comprehensiveness in the subject. ${ }^{18}$ Its results cannot be applied to clinical practice, as research questions should be formulated separately and followed by a separate analysis for each investigated event.

Therefore, the present review analyzes the effectiveness of health education to prevent nipple trauma in breastfeeding compared to other interventions.

\section{Methods}

This systematic review was conducted according to the recommendations of the Preferred Reporting Items for Systematic Reviews and Meta-Analyses (PRISMA). 19 Based on the acronym PICO, 20 the question is: is health education on breastfeeding effective to prevent nipple trauma in nursing women? In the acronym PICO, P (patient or population) refers to nursing women; I (intervention) refers to health education on breastfeeding; $\mathrm{C}$ (comparison) refers to other interventions; and $\mathrm{O}$ (outcome) refers to the prevention of nipple trauma.

In order to standardize the adopted nomenclature, interventions compared to health education on breastfeeding were referred to in this article as "nipple covers".

The research was conducted at the Universidade Federal de Goiás, campus Goiânia, GO, Brazil, from June 2018 to January 2019. Searches were conducted in the Cumulative Index to Nursing and Allied Health Literature (CINAHL), PubMed, Web of Science, and Scopus, as well as in the references cited in the selected articles.

Standardized descriptors were used from the Medical Subject Heading Section (MeSH) and the Health Sciences Descriptors (DeCS), together with non-standardized descriptors. There was no date limit for the article selection.

Two searches were performed to obtain the desired results in relation to the variable nipple trauma prevention. The search strategy comprised the following descriptors and Boolean operators:

Search 1: breastfeeding OR breast-feeding OR breast feeding AND breastfeeding technique (OR breastfeeding education OR postpartum education 
OR postnatal education OR lactation education OR postpartum positioning OR group teaching OR group education OR antenatal education) AND nipple trauma (OR nipple pain OR lactation problems).

Search 2: prevention (OR prevent OR preventing) AND breast-feeding (OR breastfeeding OR breast feeding) AND nipple trauma (OR nipple soreness OR sore nipples OR nipple fissure OR nipple cracked OR nipple cracks OR nipple cracking OR nipple crack) NOT treatment.

These search strategies resulted in 652 titles; 97 were from the Web of Science, 173 were from
PubMed, 27 were from Scopus, and 355 were from CINAHL. In addition, 12 titles were found in the list of references of the researched articles. Of the titles found, 89 were duplicated (Figure 1).

The inclusion criteria were experimental studies and publications in English, Spanish, or Portuguese. Case studies, literature reviews (narrative, integrative, and systematic), editorials, and studies that did not address the nipple trauma in nursing women were excluded.

For article selection, two reviewers independently assessedthe titles and abstracts (screening step), and subsequently the full texts (confirmation

\section{Figure 1}

Flowchart of the study selection process.
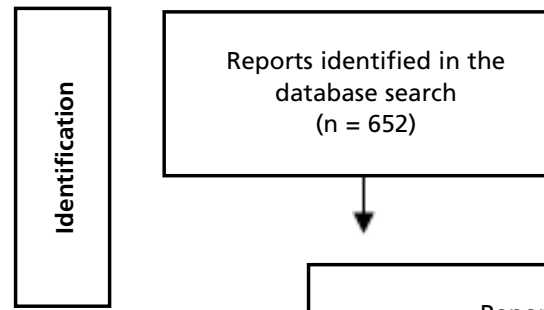

$$
(n=652)
$$

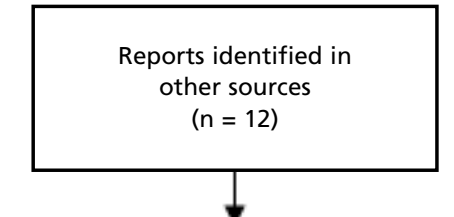

Reports after eliminating duplicates

$(n=575)$
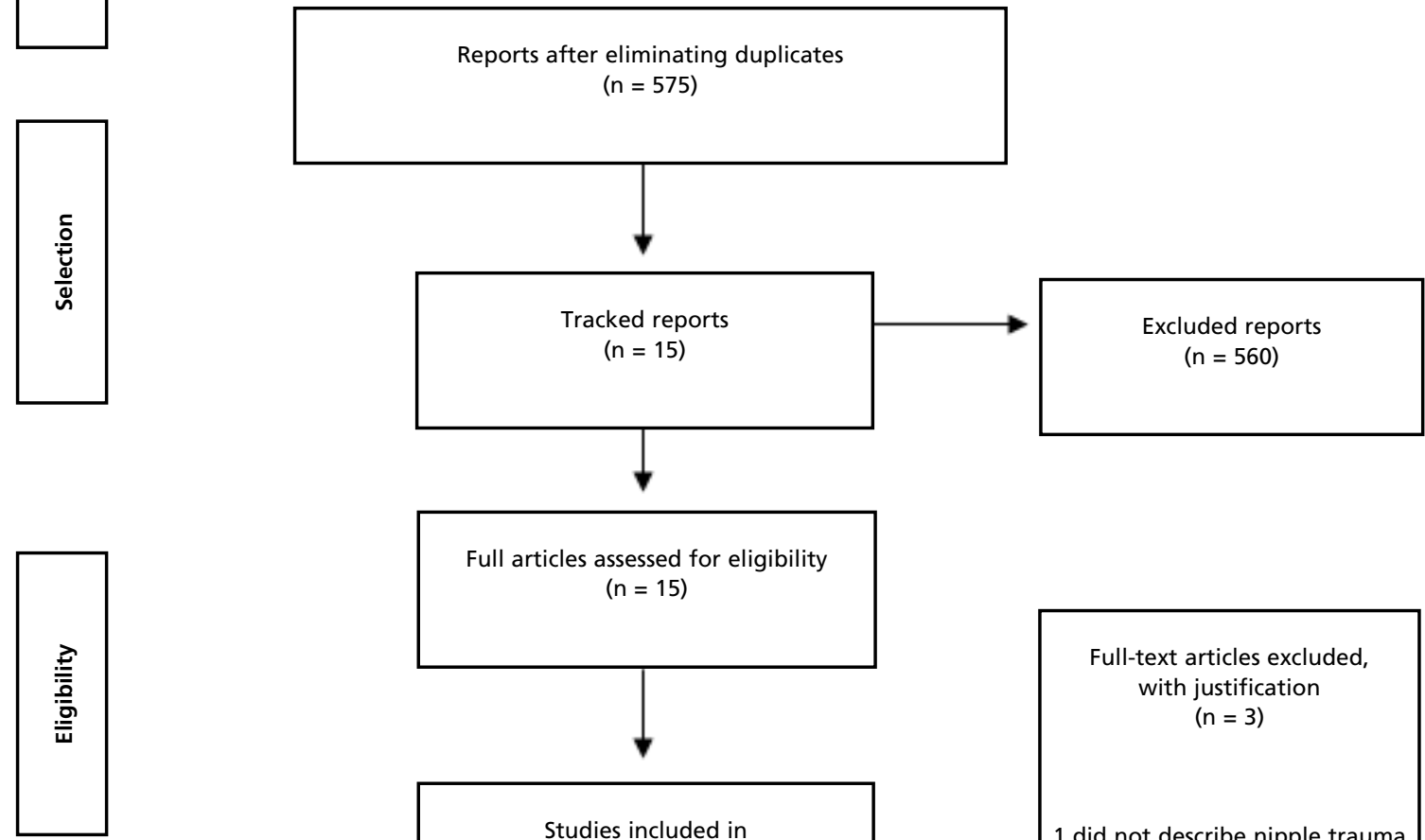

Full articles assessed for eligibility $(n=15)$
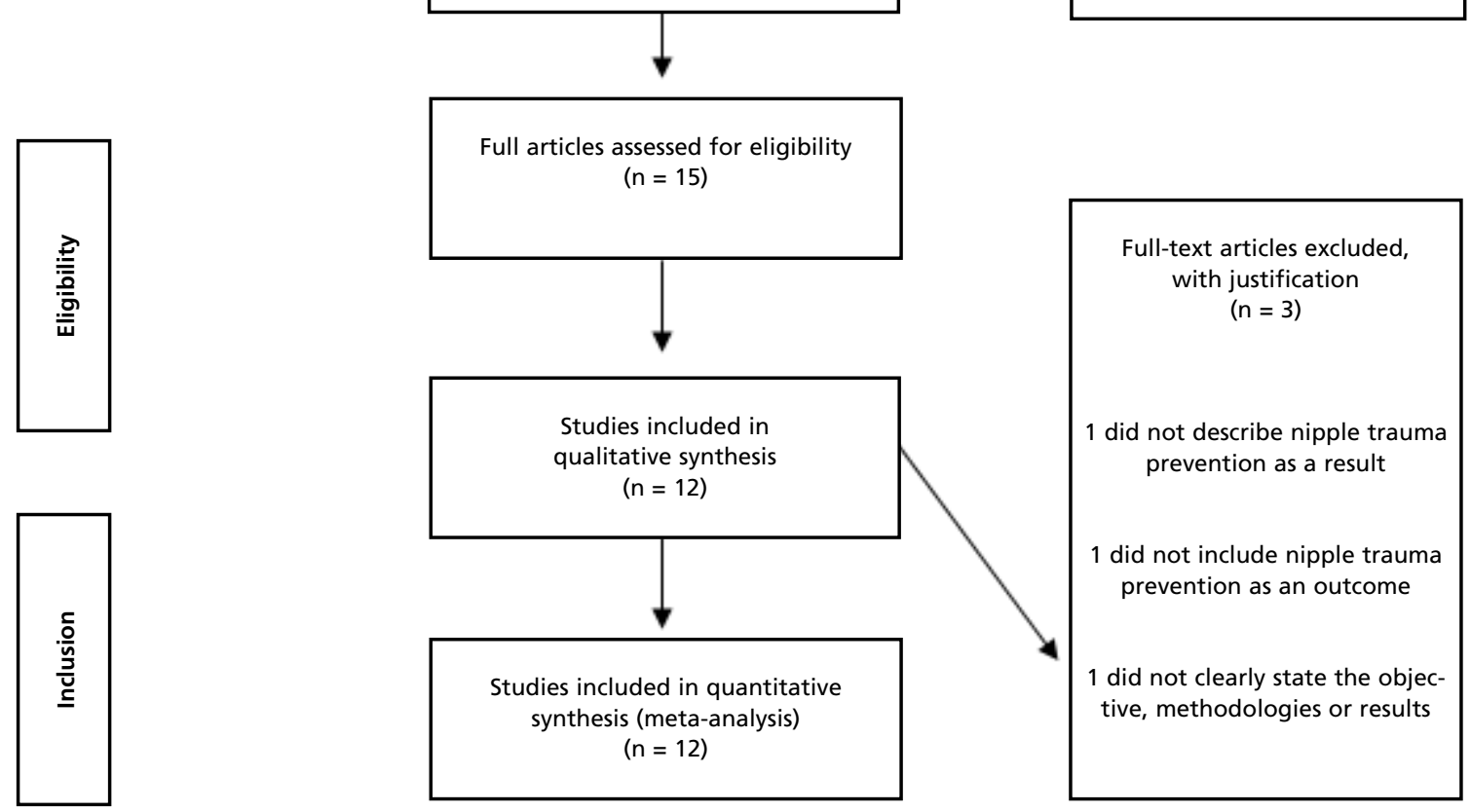
step). A third reviewer analyzed any disagreements, when necessary.

After the study selection, data were extracted by two independent reviewers and entered into a previously prepared standardized form. ${ }^{21}$ The form was used to collect general information and information on study design, characteristics of the participants, interventions, outcomes, and results.

Regarding the level of evidence, the studies were evaluated as high, moderate, or low, following the criteria established and applied in the GRADE system (Grading of Recommendations, Assessment, Development, and Evaluation). ${ }^{22}$ The studies were coded (S) and numbered sequentially from one to twelve (S1 ... S12).

\section{Results}

Tables 1 and 2 show the 12 selected studies, dating from 1987 to 2017. They were conducted in Canada $(n=1)$, Australia $(n=2)$, Italy $(n=1)$, Turkey $(n=3)$, Brazil $(n=1)$, Iran $(n=2)$, Saudi Arabia $(n=1)$, and Cuba $(n=1)$. The selected references included intervention and experimental studies, randomized clinical trial, and randomized controlled and doubleblind clinical trial. Only two studies did not use randomization (S4, S10). Samples ranged from 23 to 300 nursing mothers. All the studies were conducted at the maternities and the follow-up of the participants ranged from prenatal to six months postpartum. Three studies had no follow-up losses (S4, S11, S14). In two studies, the gestational age of the babies was not specified (S3, S12), while in the others, the babies were considered full-term. Seven of the studies included were only first-time mothers (S1, S2, S4, S8, S9, S10, S11).

Some exclusion criteria should be highlighted: abnormal nipples (flat, inverted, and pseudoinverted), $23-27$ use of pacifiers or formula, ${ }^{23-26}$ pregnancies of twins, 23-28 newborns with low birth weight, $23,24,29,30$ and Cesarean section. $25-27$

Three studies were assessed as high level of evidence (S5, S6, and S7), five as moderate (S2, S3, $\mathrm{S} 4, \mathrm{~S} 8$, and S9), and four as low (S1, S9, and S10) according to GRADE. 22

Only S2 and S12 described possible adverse events. S2 reported that the intervention may have caused anxiety in the participants, while S12 reported the absence of adverse events.

Health education on breastfeeding to prevent nipple trauma, mainly addressing the correct breastfeeding technique, was assessed directly in four studies (S2, S4, S6, S12), while health education associated with another method was assessed in fives studies (S1, S7, S8, S9, S10). Of these studies, only three described the evaluation of breastfeeding technique used by the mothers (S2, S6, S9); the other studies did not mention the care given in this regard (S3, S5, S11).

The following interventions for nipple trauma prevention were tested: health education, focusing on correct breastfeeding technique (Table 1); expressed breast milk, lanolin, guaiazulene (dark blue crystalline hydrocarbon obtained from the oil of the Guaiacum officinale tree), peppermint gel, peppermint water, gauze, tea compress, warm compress, extra-virgin olive oil, ointment based on the composition of the studied hospital (containing high-quality protein, glycerin, paraffin oil, casein, lanolin, petroleum jelly, zinc oxide, potassium hydrate, sodium and distilled water; and clostebol acetate + neomycin sulfate spray) (Table 2 ).

\section{Discussion}

\section{Health education on breastfeeding}

Health education on breastfeeding was carried out in the prenatal or postpartum periods focusing on the correct technique, has moderate evidence to prevent nipple trauma and requires to be delivered along with clinical demonstrations (S1, S4).

In the study in which breastfeeding education was provided in prenatal care, it was concluded that, to prevent nipple trauma, a one-hour session with emphasis on the correct position and grasp using a baby dummy was more effective than the regular guidelines (S1). The opportunity to experience, as close to reality as possible, helps puerperal women understand and adequately replicate breastfeeding technique. 15

Low-cost training with minimal technology was offered to 603 pregnant women in the third trimester, increased confidence regarding breastfeeding $(p=0.05)$ and provided sufficient knowledge in the postpartum period $(p=0.02)$ However, training was insufficient to increase the duration of breastfeeding or reduce breast problems. 31

Conversely, two studies, one with moderate (S2) and the other with high (S3) recommendation, showed that health education on breastfeeding in the postpartum period, with emphasis on the correct breastfeeding technique is not more effective in preventing nipple trauma than the regular guidance provided at the maternities. The hand off technique (not positioning the baby for the mother) did not reduce intercurrence, even if the educator verbally and visually described the appropriate technique with the use of teaching materials (S2). 
S1. Duffy et al.14 1997, Australia

Investigate the effect of a group teaching session for nulliparous women in the third trimester of pregnancy

Randomized experimental study with 75 primiparous women intending to breastfeed, conducted during the prenatal follow-up.

$E G *(n=37)$ : received prenatal education inter-

vention. regular prenatal educa-

Followed up to the 4th postnatal day for evaluation of breastfeeding and nipple pain and rauma (NTI)) In the 6th week the maintenance of breastfeeding was verified.

S2. Henderson et Evaluate the effectiveness of al.,28 2001, a standardized educational intervention in bre

Randomized clinical trial with 160 primiparous women intending to breastfeed.

EG $(n=80)$ : received health education on standardized and individual breastfeeding with verbal, visual, and written approach within 24 hours postpartum.

CG $(n=80)$ : received the regular care of the unit.

The groups were evaluated

3 and 6 months.

\section{S3. Oliveira et al.29 Evaluate the impact of a} 2006, Brazil breastfeeding technique intervention on the exclusive breastfeeding rate and on related breast problems in the first month postpar-

$$
\text { pairs. }
$$

Randomized clinical trial with 211 mother-child

EG ( $n=74)$ : guidelines with the use of educational material on the technique of breastfeeding.

CG $(n=137)$ : conventional guidelines of the unit.

The evaluation was performed at the maternity, at 7 th and 30 th days postpartum.
The intervention group had higher overall NTI score (M§ = $32.85, \mathrm{SD} \|=5.5$ ), indicating less nipple trauma $p<0.001$;
Moderate

There was no significant difference between the groups in the incidence of nipple trauma.

Moderate
Practice training delivered to prenatal group effectively reduced nipple pain and trauma in nulliparous tramen ind increased breastfeeding rates.
There was no significant difference $(p>0.05)$ between th groups regarding the frequency of nipple trauma in
the maternity hospital $(E G=$ $43.2 \% \times \mathrm{CG}=43.8 \%)$, on the 7 th day ( $E G=43.2 \% x$ $\mathrm{CG}=48.9 \%$ ) and the 30th day ( $E G=8.5 \% \times C G=9.1 \%)$.
One session in the maternity hospital to reinforce the proper breastfeeding technique was insufficient to improve the breastfeeding technique or reduce the occurrence of breast problems resulting from poor technique. 
Characteristics of the studies included in the systematic review on the use of health education in preventing nipple trauma.

$\begin{array}{llll}\text { Author, Year, Country } & \text { Proposal } & \text { Method } & \text { Outcome }\end{array}$

Controlled intervention study with 90 primiparous women.

S4. Eksioglu et al.15 Investigate the effects of dif2017, Turkey ferent pre-discharge breastfeeding training techniques for primiparous mothers on the subsequent incidence of nipple trauma.

$\mathrm{G} 2(\mathrm{n}=30)$ : provision of package leaflet.

$\mathrm{G} 3(\mathrm{n}=30)$ : demonstration-based training.

In the $2^{\text {nd }}$ week postpartum the rate of nipple trauma was $63.3 \%$ in $\mathrm{G} 1,56.7 \%$ in $\mathrm{G} 2$, and $20.0 \%$ in $\mathrm{G} 3(p=0.001)$.

In the $4^{\text {th }}$ week postpartum,

the rate of nipple trauma was

$30.0 \%$ in $\mathrm{G} 1,10.0 \%$ in $\mathrm{G} 2$,

${ }^{\star} \mathrm{EG}=$ experimental group; ${ }^{\mathrm{C}} \mathrm{CG}=$ control group; ${ }^{\mathrm{N} T \mathrm{TI}}=$ nipple trauma index; $\$ \mathrm{M}=$ mean; $\| \mathrm{SD}=$ standard deviation. 
S5. Hewat et al.38 Evaluate whether breast milk 1987, Canada or colostrum, applied to the nipples after breastfeeding, is superior to hydrated lanolin in preventing nipple pain and trauma
Randomized clinical trial with 23 primiparous women evaluated until the $10^{\text {th }}$ day postpar-

$$
\text { tum. }
$$

G1 ( $n=10)$ applied expressed breast milk or the left after each feeding.

$2(n=13)$ reversed the procedure.

Each participant was her own control.

56. Centuori et al.30 Determine whether the dura1999, Italy tion of breastfeeding and the incidence of nipple trauma and pain are affected by the use of ointments and creams.

57. Api et al.25 Evaluate the effectiveness of 2005, Turkey guaiazulene cream compared to breast milk in the preven tion of nipple pain and trau-
ma during breastfeeding.

double-blind clinica trial with women of vaginal delivery in the puerperium.

EG ( $n=76)$ : used guaiazulene ointment $0.05 \%$ after each feeding. Breast cleaning was recom mended before feeding

CG ( $n=77)$ : used breast milk after each feeding. The women were evaluated 15 and 30 days after delivery for nipple pain and trauma.
All the women experienced nipple trauma.

There was a positive and significant correlation between nipple trauma and engorgement $(r=0.43 ; p<0.02)$.
Low

Breast milk was not superior to lanolin in the prevention or reduction of nipple trau$\mathrm{ma}$ in the first 10 days postpartum.
There was no difference between the two groups regarding breast and nipple problems.

Bottle and pacifier use were associated with nipple trauma discharge and two weeks postpartum.

The total incidence of nipple trauma was $31.4 \%$ at 30 days postpartum, $18.4 \%$ in $\mathrm{EG}$ and $44.2 \%$ in CG $(p=0.001$, $\left.\mathrm{RR}^{\ddagger}=0.42, \mathrm{C} \mid 95 \%=0.24-0.71\right)$.
Moderate Special cleaning with distilled water and sterile gauze, and the use of creams and spray on the nipples proved to be unnecessary. nipple trauma.

${ }^{*} \mathrm{EG}=$ experimental group; ${ }_{\mathrm{H}} \mathrm{CG}=$ control group; $¥ \mathrm{RR}=$ relative risk; $\mathrm{EVOO}=$ extra virgin olive oil. 
Table 2

Characteristics of the studies included in the systematic review on the use of nipple covers in preventing nipple trauma.

\begin{tabular}{llll}
\hline Author, Year, Country & Proposal & Method & Outcome
\end{tabular}

Level of evidence

Conclusion

58. Melli et al.23

2007, Iran

Investigate the preventive effect of peppermint gel on nipple trauma, compared to modified lanolin and placebo

primiparous women evaluated on days $4,7,14$, and 42 postpartum.

1 $(n=72)$ : purified lanolin

G2 $(n=72)$ : peppermint gel.

G3 ( $n=72)$ : placebo gel.

The intervention was applied to the areola and nipple after each feeding and cleaned before

the next feeding. All the women received

guidelines on breastfeeding prior to breast-

feeding.

59. Melli et al.24 Evaluate the effectiveness of

a topical preparation of peppermint water in comparison with expressed breast milk for the prevention of nipple trauma in nursing primiparous women.

Randomized clinical trial with 196 women, followed-up in three visits or phone calls within 14 days and one phone call within the sixth week postpartum.

EG ( $n=98)$ : application of peppermint water after each feeding

CG ( $n=98)$ : application of breast milk after each feeding.

All mothers received standardized breastfeed-

ing education with demonstrations before starting breastfeeding.

S10. Atan e Sirin.27 Compare the effectiveness of 2012, Turkey breast milk, warm wet com-
press, and tea compress in the prevention of nipple the prevention of nipple
problems in primiparous women.
Experimental study with 105 primiparous women.

G1 $(n=35)$ : tea compress

$2(n=35)$ : warm wet compress G3 $(n=35)$ : breast milk

Health education on the benefits and success of breastfeeding was provided, together with a brochure to support this education. Visits on the $5^{\text {th }}$ and $14^{\text {th }}$ day postpartum.
Nipple trauma was less common when using peppermint gel

(3.8\%) than when using lanolin ointment $(6.9 \%)$ or placebo day postpartum.

The relative risk of trauma in the lanolin group ( $R R=2.41$, $\mathrm{Cl} 195 \%=1.20-3.01)$ was higher than in the ( $R=1.85$, Cl95\%=1.64-3.10).

The occurrence of nipple trauma was lower in the group using peppermint water $(p<0.01) \mathrm{EG}=7 \%, \mathrm{CG}=23 \%$. In the EG, trauma occurred on the 7th day postpartum (6.2 1.9 ) and in the $\mathrm{CG}$, on the $4^{\text {th }}$ day postpartum $(3.8 \pm 0.9)$. Peppermint water reduced the risk of trauma compared to the other group $(R R=3.6$, $\mathrm{C} \mid 95 \%=2.9,4.3)$.

The highest rate of nipple trauma $(34.3 \%)$ occurred on the 3 rd day postpartum.

On the 6 th and 7 th days postpartum, fewer nipple problems were identified in the warm wet compress group ( $p>0.023$ ). On the 14th day postpartum, there was no significant difference between the groups, $\mathrm{p}=609$ ( G1 $=54.3 \%$, G2=42.9.
High

Peppermint gel formulated as a natural remedy was effective in preventing nipple trauma. The application of the gel was suggested as prophylaxis for nipple trauma with proper instruction at the start of breastfeeding.

Moderate

Peppermint water was effective in preventing nipple pain and trauma compare with breast milk.
Midwives and nurses can teach mothers in the hospital about warm wet compresses to prevent nipple pain and trauma and continue the practice after discharge. 
Characteristics of the studies included in the systematic review on the use of nipple covers in preventing nipple trauma.

Author, Year, Country Proposal

S11. Thabet et al.26

2013, Saudi Arabia

Compare the effect of peppermint water compared to breast milk for the prevention of nipple injuries.

Experimental study with 150 primiparous women breastfeeding after vaginal delivery with 38 or more weeks of gestation, from the 1 st to the 14 th day postpartum.

$G 1(n=50)$ : peppermint water applied after each feeding with a gauze on the nipples and areo-

las after washing the nipples with water.

G2 $(n=50)$ : expressed breast milk after each feeding.

CG $(n=50)$ : nipples were kept clean and dry. All the women received comprehensive hospital education on breastfeeding before the start of breastfeeding.

S12. Cordero et al.40 Evaluate the effectiveness of 2015, Cuba extra virgin olive oil (EVOO) in the relief and prevention of sore nipples in nursing mothers compared to the use of breast milk.

Experimental, prospective, randomized trial with 300 nursing mothers.

G1 (n=150): EVOO on the nipple after each

$$
\text { feeding }
$$

rer each feeding. Clinical evaluations were performed in the first two weeks of treatment and after the first month of breastfeeding.
Women in the peppermint areolas at 15 and 30 days $(88 \%$ and $92 \%)$ than women in the breast milk group $(60 \%$ and $64 \%)$ and CG $(44 \%$ and $40 \%)(p<0.001)$.
Low

Peppermint water was more effective in preventing nipple trauma and pain than tions at the beginning of the breastfeeding program are required.
The occurrence of nipple trauma in the EVOO group: $2.7 \%$.

In the group that used breast milk: $44.0 \%$.

Nipple trauma was related to poor posture during feeding $(p<0.001)$
Low

EVOO was important in the prevention of nipple trauma, with a positive result even during feeding.

${ }^{\star} \mathrm{EG}=$ experimental group; ${ }^{\top \mathrm{CG}}=$ control group; ${ }_{\mathrm{R} R}=$ relative risk; $\mathrm{EVOO}=$ extra virgin olive oil. 
In $\mathrm{S} 2$, late evaluations regarding the presence or absence of nipple trauma may have contributed to the non-difference observed between experimental and regular education, since the nipple trauma could have healed naturally in week six, month three, and month six postpartum, albeit slowly. Ideally, the evaluation should be done in the first week, since this event occurs between the $2^{\text {nd }}$ and $3^{\text {rd }}$ day postpartum. ${ }^{8}$ Late evaluation may also have affected the follow-up of the intervention by the postpartum woman, who could have sought, on her own, some treatment or nipple cover to relieve pain and trauma, or could even have stopped breastfeeding due to pain.

In $\mathrm{S} 3$, the regular guidance was delivered by health workers at a maternity hospital accredited by the program known as Iniciativa Hospital Amigo da Criança (Child-Friendly Hospital Initiative), where the entire team is skilled to support breastfeeding. ${ }^{1}$ This initiative has a positive impact on the breastfeeding process, 32 which would explain the nondifference observed between the regular guidance and the experimental guidance delivered by the team specialized in breastfeeding.

The group mentioned above had a reduced rate of nipple trauma $(8.5 \%$ in the experimental group and $9.1 \%$ in the regular group) on the $30^{\text {th }}$ day postpartum. In another study (S7), however, a nipple cover was considered effective in preventing trauma, with a rate of $31.4 \%$ in the equivalent postpartum period. This result suggests that the protective effect lies in health education.

On the other hand, there is a strong recommendation that points out that a single intervention is insufficient to ensure an adequate technique during feeding (S3). Even with individual guidance, the breastfeeding technique used in the first month changes little in relation to quality, regardless of whether the mother has received the intervention. 28

Inadequate position and latch during feeding are the main causes of nipple trauma 13,33 and, consequently, causes persistent nipple pain, ${ }^{34}$ usually of high intensity. 35

Thus, health education on breastfeeding, whether individual or in group, focuses on the proper breastfeeding technique that should be routinely provided in the prenatal and postpartum period from the first feeding, ${ }^{36}$ with a follow-up of at least two visits. ${ }^{37}$

Puerperal women who receive demonstrationbased training have higher positive scores for use of the correct feeding technique than the puerperal women of the other groups $(p<0.05)$. This training is based on explaining, demonstrating, and practicing breastfeeding with a breast model, dolls, and illus- trative guide, and lasts for approximately one hour (S4).

\section{Use of nipple covers}

Breast milk was tested in half of the studies (S5, S7, S9, S10, S11, S12) and was reportedly less effective than guaiazulene (S7), peppermint water (S9, S11), warm wet compress (S10), and extra virgin olive oil (S12), while no difference was shown with lanolin (S5).

In Hewat and Ellis ${ }^{38}$ study revealed that breast milk was not superior to lanolin in preventing nipple pain and trauma. However, this study has a low level of evidence due to its methodological weaknesses: 1. The small sample size did not allow the use of parametric procedures to determine the statistical differences between the two interventions; 2 . Variables related to the breastfeeding technique, which could affect nipple pain and trauma, were not controlled; and, 3. The woman herself was her control, which may have caused confusion or forgetfulness at the time of applying milk or lanolin, resulting in a strong research bias.

In contrast, the experimental study conducted by Thabet et al. 26 showed that breast milk applied to the nipple and areola was less effective than just keeping this region clean and dry (S11). The relative risk of nipple and areola trauma in the control group (48\% and $56 \%$ ) and in the expressed breast milk group (32\% and $32 \%$ ) was higher than in the peppermint water group ( $4 \%$ and $2 \%$ ) at 15 and 30 days after delivery, with statistical significance.

S6, with a moderate recommendation, points out that avoiding any intervention is better than the nipple cover prepared by the hospital. Although there was no significant difference, the rate of nipple trauma at hospital discharge was higher in the group that used the hospital nipple cover than in the group that avoided any intervention (34\% and $27 \%)$.

Although S6 scope did not include evaluating the breastfeeding technique, it suggests that breastfeeding guidance and support may be more effective in reducing nipple problems, in addition to avoiding unnecessary spending on nipple covers. ${ }^{30}$

The guaiazulene cover showed better results than breast milk. Despite being a high-evidence study, $31.4 \%$ of women still had nipple pain and trauma at 30 days postpartum. This result can be explained by the fact that the interventions were conducted by a health worker with no experience in breastfeeding and the appropriate breastfeeding technique was not included in the intervention. In addition, guaiazulene is an unconventional nipple cover for breastfeeding women and it must be removed 
before feedings.

Therefore, it is suggested that, before its widespread use in clinical practice, a randomized clinical trial should be conducted to compare guaiazulene with other nipple covers with effective results in preventing nipple trauma. Moreover, guidelines and evaluations on the correct breastfeeding technique should be provided for all groups, with an additional focus on cost-effectiveness. ${ }^{9}$

Another nipple cover with a positive effect was peppermint gel, with high recommendation (S8), and peppermint water, with moderate (S9) and low recommendation (S11).

The results show that peppermint gel, formulated as a natural treatment, is more effective in preventing nipple trauma than lanolin (S8), placebo gel (S8), and breast milk (S9, S11). The rate of nipple trauma on the 14th postpartum day was lower in the study (S8) that used gel $(3.8 \%)$ than in the study (S9) that used peppermint water $(9.0 \%)$. Still, peppermint water was three times more effective than expressed breast milk (27\%) (S9).

Peppermint (Mentha piperita) has a calming and numbing effect that could explain the relief in skin irritations of the areolomamillary region.23,24,26 It also has an antiseptic effect and increases the flexibility of tissue, making it resistant to injuries. 23

Peppermint gel can be suggested as prophylaxis for nipple trauma, together with adequate health education at the beginning of breastfeeding. The formula for the preparation is available in S8. Additionally, the gel is easier to apply than peppermint water.

Noteworthily, there is a need to remove peppermint gel and peppermint water before feedings. The standardization of removal for all groups may have impaired the performance of lanolin (S8) since pure lanolin does not require removal. Moreover, lanolin has had positive effects in the treatment of nipple pain and trauma, ${ }^{39}$ and should be further investigated for the prevention of these conditions.

The removal of nipple covers (S6, S7, S8, S9) for breast hygiene before each feeding can weaken the skin of the areolomamillary region, making it susceptible to trauma or even worsen existing injuries. It is recommended that rubbing should be avoided and only water be used to clean the region, during daily baths, without the use of soap or other astringent products. ${ }^{1}$

Extra virgin olive oil was recently tested (S12), and it was found that, at 15 days postpartum, only $2.7 \%$ of women had nipple trauma compared to $44 \%$ of the women who used expressed breast milk. It is believed that this effectiveness is due to the anti- inflammatory and antioxidant action of extra virgin olive oil. However, this is considered a study with a low level of evidence. 40

Study S12 assigns extra virgin olive oil protective action for trauma even when the feeding technique is inadequate; however, it does not include an inferential analysis to identify a possible difference between the groups. A poor breastfeeding technique was observed in $36.9 \%$ of the women in the breast milk group and in $18.7 \%$ of the women in the olive oil group. An inferential analysis made with the available data unveils a significant difference between the groups; i.e., the group using breast milk exhibited a worse breastfeeding technique, which would justify a higher rate of nipple trauma.

In addition, the authors do not clarify which follow-up or guidelines were provided to the groups on breastfeeding, especially in relation to the proper breastfeeding technique. It is wondered whether there was differentiation between the groups or whether the group using extra virgin olive oil received more follow-up and guidelines on breastfeeding and proper techniques.

Women, pregnant women or in the puerperium, need to feel supported and accompanied by a health worker trained in breastfeeding, even by phone calls with consistent information about breastfeeding. 41,42

Therefore, health education on breastfeeding should be provided in more than one contact with the mothers, $29,37,42$ to improve the knowledge and attitudes of women regarding prevention of nipple trauma.23,24,26 These contacts should preferably occur during the prenatal period, $27,28,29,36,38$ with active participation of the women in demonstrations of the breastfeeding technique. 15 The support provided by health workers should involve health education on breastfeeding. It may include a nipple cover with moderate to high level of evidence; ideally one that does not require removal, such as lanolin, 7,17 still minimally explored in clinical studies on trauma prevention. ${ }^{23,38}$

A limitation of this study is the absence of a meta-analysis, which would be impracticable due to the diversity of nipple covers and the non-uniformity of the strategies the studies applied to interventions. Moreover, a knowledge gap was identified regarding experimental or quasi-experimental studies conducted in prenatal care, since almost all clinical studies in preventing nipple trauma were conducted in the postpartum and in the maternity hospital.

Health education on breastfeeding, with clinical demonstration, has a moderate level of evidence in preventing nipple trauma. In contrast, the single approach of health education in the postpartum 
period does not effectively prevent nipple trauma.

Interventions with peppermint gel or water and guaiazulene covers have a positive effect in preventing nipple trauma, with high or moderate recommendation, in the postpartum period. The use of these nipple covers in the clinical practice of health workers involved with breastfeeding needs to be considered, since all require the removal before feeding. It is not possible to recommend the use of extra virgin oil in preventing nipple trauma due to the methodological weaknesses of the study.

Comparative studies on health education with isolated clinical demonstration versus education combined with nipple covers, both prenatal and postpartum, are required to establish whether the prevention of trauma is related to covers or to a well applied health education.

\section{References}

1. World Health Organization, UNICEF. Implementation guidance: protecting, promoting and supporting breastfeeding in facilities providing maternity and newborn services: the revised baby-friendly hospital initiative. Geneva: World Health Organization; 2018.

2. Chowdhury R, Sinha B, Sankar MJ, Taneja S, Bhandari N, Rollins N, Bahl R, Martines J. Breastfeeding and maternal health outcomes: a systematic review and meta-analysis. Acta Paediatr. 2015; 104 (467): 96-113.

3. UNICEF. Breastfeeding: amother's gift, for every child. New York: UNICEF; 2018

4. Victora CG, Barros AJD, França GVA, Bahl R, Rollins NC Horton S, et al. Breastfeeding in the 21 st century: epidemiology, mechanisms, and lifelong effect. Lancet. 2016; 387 (10017): 475-90.

5. Vieira GO, Martins CC, Vieira TO, de Oliveira NF, Silva LR. Factors predicting early discontinuation of exclusive breastfeeding in the first month of life. J Pediatr. 2010; 86 (5): 441-4.

6. Moraes BA, Gonçalves AC, Strada JKR, Gouveia HG Factors associated with the interruption of exclusive breastfeeding in infants up to 30 days old.Rev Gaúcha Enferm. 2016;37: 2016-44

7. Abou-Dakn M, Fluhr JW, Gensch M, Wöckel A. Positive effect of HPA lanolin versus expressed breastmilk on painful and damaged nipples during lactation. Skin Pharmacol Physiol. 2011; 24 (1): 27-35.

8. Vieira F, Mota D, Castral TC, Guimaraes JV, Salge AKM, Bachion MM. Effects of Anhydrous Lanolin versus Breast Milk Combined with a Breast Shell for the Treatment of Nipple Trauma and Pain During Breastfeeding: A Randomized Clinical Trial. J Midwifery Womens Health. 2017; 62 (5): 572-9.

9. Dias JS, Vieira TO, Vieira GO. Factors associated to nipple trauma in lactation period: a systematic review. Rev Bras Saúde Matern Infant. 2017; 17 (1): 27-42.

\section{Authors' contribution}

Oliveira FS and Vieira F participated in the study design, selection of articles, interpretation and discussion of data, as well as in the writing of the article. Cecilio JO selected the articles to be included in the study. Guimaraes JV and Campbell SH conducted a critical review of the manuscript. All authors approved the final version of the article.

10. Thompson R, Kruske S, Barclay L, Linden K, Gao Y, Kildea S. Potential predictors of nipple trauma from an inhome breastfeeding programme: A cross-sectional study. Women Birth. 2016; 29 (4): 336-44.

11. Ingram J, Jhonson D, Copeland M, Taylor HJ. The development of a new breast feeding assessment tool and the relationship with breast feeding self-efficacy. Midwifery. 2015; 31 (1): $132-7$

12. Vieira F, Bachion MM, Mota DD, Munari DB. A systematic review of the interventions for nipple trauma in breastfeeding mothers. J Nurs Scholarsh. 2013; 45 (2): 116-25.

13. Cirico MOV, Shimoda GT, de Oliveira RNG. Healthcare quality in breastfeeding: implementation of the nipple trauma index. Rev Gaúcha Enferm. 2016; 37 (4): e60546.

14. Duffy EP, Percival P, Kershaw E. Positive effects of an antenatal group teaching session on postnatal nipple pain, nipple trauma and breast feeding rates. Midwifery. 1997; 13 (4): 189-96.

15. Eksioglu A, Yesil Y, Gungor DD, Turfan EC. The effects of different breastfeeding training techniques for primiparous mothers before discharge on the incidence of cracked nipples. Breastfeed Med. 2017; 12 (5): 311-5.

16. Vasquez J, Dumith SC, Susin LRO. Maternal breastfeeding: a comparative study of knowledge and management among Family Health Strategy professionals and those using the traditional model.Rev Bras Saúde Matern Infant. 2015; 15 (2): $181-92$.

17. Costa AA, Souza EB, Guimarães JV, Vieira F. Evidências das intervenções na prevenção do trauma mamilar na amamentação: revisão integrativa. Rev Eletr Enf. 2013; 15 (3): 790-801.

18. Niazi A, Rahimi VB, Soheili-Far S, Askari N, RahmanianDevin P, Sanei-Far Z,Sahebkar A, Rakhshandeh H, Askari VR. A Systematic Review on Prevention and Treatment of Nipple Pain and Fissure: Are They Curable?.J Pharmacopuncture. 2018; 21 (3): 139-50. 
19. Moher D, Shamseer L, Clarke M, Ghersi D, Liberati A, Petticrew M, Stewart LA. Preferred reporting items for systematic review and meta-analysis protocols (PRISMAP) 2015 statement. Syst Rev. 2015; 4: 1 .

20. Santos CMC, Pimenta CAM, Nobre MRC. The PICO strategy for the research question construction and evidence search. Rev Latino-Am Enferm. 2007;15(3):508-11.

21. UNiversity of York. Centre for Reviews and Dissemination. Systematic reviews: CRD's guidance for undertaking reviews in health care. University of York: Centre for Reviews \& Dissemination; 2009.

22. GRADEpro GDT: GRADEpro Guideline Development Tool [Software]. McMaster University, 2015 (developed by Evidence Prime, Inc.). Available from: gradepro.org.

23. Melli MS, Rashidi MR, Nokhoodchi A, Tagavi S, Farzadi L, Sadaghat K, Tahmasebi Z, Kazemi AM. A randomized trial of peppermint gel, lanolin ointment, and placebo gel to prevent nipple crack in primiparous breastfeeding women. Med Sci Monit. 2007; 13 (9)

24. Melli MS, Rashidi MR, Delazar A, Madarek E, Maher MHK, Ghasemzadeh A, Sadaghat K, Tahmasebi Z. Effect of peppermint water on prevention of nipple cracks in lactating primiparous women: a randomized controlled trial. Int Breastfeed J. 2007; 2 (7).

25. Api M, Sivri D, Api O, Görgen H, Çetin A, Yayla M Prevention of Nipple Cracks With Guaiazulene Versus Breast Milk in Nursing Mother: A Randomized, Controlled, Double-Blind Trial. J Turkish German Gynecol Assoc. 2005; 6 (4): 279-84

26. Thabet HA, Mourad MA, Alahadal AM, Alsenany S, Alsaif A. Prevention of Nipple Cracks with Peppermint Water versus Breast Milk in Lactating Primiparous Women. Life Science J. 2013;10(4): 2010-7. Disponível em: http://www.lifesciencesite.com/lsj/life1004/266_21318life1 004_2010_2017.pdf

27. Atan ŞÜ, Sirin A. Prevention of nipple problems in primipara breastfeeding mothers: A pilot study. Healthmed. 2012; 6 (12): 4258-66

28. Henderson A, Stamp G, Pincombe J. Postpartum positioning and attachment education for increasing breastfeeding: a randomized trial. Birth. 2001; 28 (4): 236-42.

29. Oliveira LD, Giugliani ER, Espirito Santo LC, França MC, Weigert EM, Kohler CV, Lourenzi Bonilha AL. Effect of intervention to improve breastfeeding technique on the frequency of exclusive breastfeeding and lactation-related problems. J Hum Lact. 2006; 22 (3): 315-21.

30. Centuori S, Burmaz T, Ronfani L, Fragiacomo M, Quintero $\mathrm{S}$, Pavan C, et al. Nipple care, sore nipples, and breastfeeding: a randomized trial. J Hum Lact. 1999;15(2):12530 .

Received on February 15, 2019

Final version presented on November 1, 2019

Approved on January 3, 2020
31. Kronborg H, Maimburg RD, Vaeth M. Antenatal training to improve breast feeding: a randomised trial. Midwifery. 2012; 28 (6): 784-90

32. Zarshenas M, Zhao Y, Binns CW, Scott JA. Baby-friendly hospital practices are associated with duration of full breastfeeding in primiparous but not multiparous Iranian women. Matern Child Nutr. 2018; 14 (3): e12583.

33. Puapornpong P, Paritakul P, Suksamarnwong M, Srisuwan S, Ketsuwan S. Nipple Pain Incidence, the Predisposing Factors, the Recovery Period After Care Management, and the Exclusive Breastfeeding Outcome. Breastfeed Med. 2017; 12: 169-73

34. Buck ML, Amir LH, Cullinane M, Donath SM. Nipple Pain, Damage, and Vasospasm in the First 8 Weeks Postpartum. Breastfeed Med. 2014; 9 (2): 56-62.

35. Coca KP, Amir LH, Dos Remedios da Silva Alves M, Barbieri M, Marcacine KO, Vilhena Abrao ACF. Measurement tools and intensity of nipple pain among women with or without damaged nipples: A quantitative systematic review. J Adv Nurs. 2019; 75 (6): 1162-72.

36. Karaçam Z, Sağlık M. Breastfeeding problems and interventions performed on problems: Systematic review based on studies made in Turkey. Turk Pediatri Ars. 2018; 53 (3): 134-48.

37. Tiruye G, Mesfin F, Geda B, Shiferaw K. Breastfeeding technique and associated factors among breastfeeding mothers in Harar city, Eastern Ethiopia. Int Breastfeed J. 2018; 13: 5 .

38. Hewat RJ, Ellis DJ. A comparison of the effectiveness of two methods of nipple care. Birth. 1987; 14 (1): 41-5.

39. Mariani Neto C, Albuquerque RS, Souza SC, Giesta RO, Fernandes APS, Mondin B. Comparative study of the use of HPA Lanolin and breast milk for treating pain associated with nipple trauma. Rev Bras Ginecol Obstet. 2018; 40: 664-72.

40. Cordero MJA, Villar NM, Barrilao RG, Cortés MEC, López AM. Application of Extra Virgin Olive Oil to Prevent Nipple Cracking in Lactating Women. Worldviews on Evid Based Nurs. 2015; 12 (6): 364-9.

41. Fu IC, Fong DY, Heys M, Lee IL, Sham A, Tarrant M. Professional breastfeeding support for first-time mothers: a multicentre cluster randomised controlled trial. BJOG. 2014; 121(13): 1673-83

42. Howell EA, Bodnar-Deren S, Balbierz A, Parides M, Bickell N. An intervention to extend breastfeeding among black and Latina mothers after delivery. Am J Obstet Gynecol. 2014; 210 (3): 239. 